\title{
Common Presentation of an Uncommon Disease- Strongyloidiasis could be Devastating in an Immunocompromised Host
}

\author{
Mohammad Shohidul Islam*, Sharmin Tahmina Khan, Arunangshu Raha, Farid Ahmed, Mir Jakib Hossain, Faruque Ahmed \\ Department of Medical Gastroenterology, Sheikh Russel National Gastroliver Institute and Hospital, Bangladesh
}

${ }^{*}$ Correspondence to: Mohammad Shohidul Islam, Department of Medical Gastroenterology, Sheikh Russel National Gastroliver Institute and Hospital, Dhaka, Bangladesh; Tel: 8801818711523; E-mail: msironyk56@gmail.com

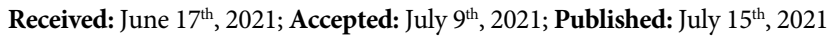

Citation: Islam MS, Khan ST, Raha A, Ahmed F, Hossain MJ, Ahmed F. Common presentation of an uncommon disease-strongyloidiasis could be devastating in an immunocompromised host. Gastro Open A Open J. 2021; 2(1): 47-49. doi: 10.33169/gastro.GOAOJ-2-111

\begin{abstract}
Strongyloidiasis is a chronic self-limited parasitic infection associated with mostly nonspecific sign symptoms. But in immunocompromised patient's morbidity and mortality is high due to hyperinfection syndrome and disseminated disease. So early diagnosis is very important. Here we report a case of hyperinfection with Strongyloides stercoralis in an elderly immunocompromised patient diagnosed by endoscopic biopsy who was treated successfully later on.
\end{abstract}

Keywords: Strongyloidiasis; parasitic infection; Immunocompromised host, Hyperinfection Disseminated disease.

\section{INTRODUCTION}

Strongyloidiasis is a parasitic disease caused by the Strongyloides stercoralis, an intestinal nematode of humans. ${ }^{1}$ It is estimated that about 30 to 100 millions of people are infected worldwide, although no precise estimate is available. ${ }^{2}$ Endemic region for infection include the Southeastern United States, Eastern Europe, Southeastern Asia, Bangladesh, Pakistan, sub-Saharan Africa, the West Indies and South America. ${ }^{3}$ The clinical symptoms and signs reported are frequently nonspecific. ${ }^{4}$ It is a chronic and self-limited disease; however, in immunocompromised patients, hyperinfection syndrome and disseminated disease can occur. ${ }^{5}$ So early diagnosis is very important, as a high rate of mortality and morbidity is associated with hyperinfection or disseminated disease. ${ }^{5-7}$

\section{CASE PRESENTATION}

A 70-year-old man, farmer, from Rupgonj, Narayangonj was admitted into SRNGIH with the complaints of vomiting, anorexia and significant weight loss for 2 months. Vomiting usually occurred 30 minutes to 40 minutes after taking meal, copious in amount, contained partially digested food materials but did not contain any blood or bile. It was not associated with any abdominal pain, distension, visible abdominal lump or constipation. He also complained of early satiety, bloating, post prandial abdominal fullness and nausea associated with vomiting. Previously he was admitted in a local hospital \& was treated conservatively with intravenous fluids, antibiotic, antiemetic and PPI. As his condition was not improving, he was transferred to SRNGIH for better management. He had no history of fever, cough, night sweats, alteration of bowel habit, jaundice, NSAID intake, hematemesis, melena, joint pain, oral ulcerations or contact with known TB patients. He had no significant past medical or surgical illness. All his family members were in good health.

On query he mentioned that four months prior to admission he was diagnosed as a case of bullous pemphigoid and was prescribed oral prednisolone by a dermatologist. After taking 3 months he stopped prednisolone by himself due to appearance of presenting symptoms.

On examination, he was ill looking, cachectic, smooth shiny tongue, mildly anemic, pitting leg edema, multiple depigmented skin patches mostly over the trunk and appeared dehydrated with normal vital signs. Systemic examination, including abdominal examination, was unremarkable. On the basis of presenting symptoms and examination findings, clinical diagnosis of gastric outlet obstruction due to carcinoma stomach was considered.

We did routine investigations and reviewed the investigations previously done. His complete blood count revealed Hb-10 gm/ dL with normal WBC count. PBF showed dimorphic anemia. He had hyponatremia and hypokalemia. Serum Albumin was $21 \mathrm{gm} / \mathrm{L}$. Chest 
X-ray and USG of whole abdomen was normal. Endoscopy of upper GIT was done which revealed patchy erythema, erosions at body and antrum of the stomach. Duodenal bulb was deformed and partially narrowed. Mucosa of the first part of the duodenum showed nodularity, friability, marked erythema and edema. (Figure 1) Biopsy was taken from the bulb of the duodenum and histopathology report revealed nematode infestation with chronic duodenitis. (Figure 2) Fresh stool sample was sent for routine microscopic examination and revealed many larvae of strongyloides stercoralis.

Figure 1: Endoscopy of upper GIT showing patchy erythema and crosions at stomach. Duodenal bulb is deformed and partially narrowed. Mucosa of the D1 shows nodularity and friability.

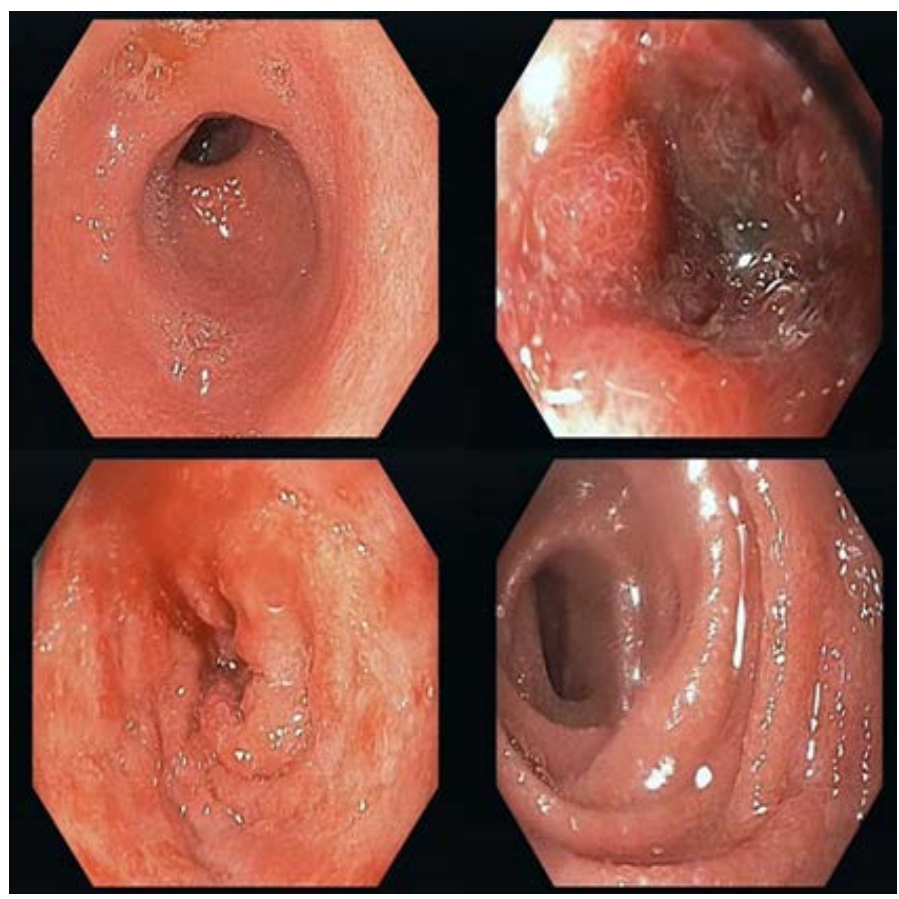

Figure 2: Histopathology of duodenal mucosa showing cross section of strongyloids stercoralis within the crypts.

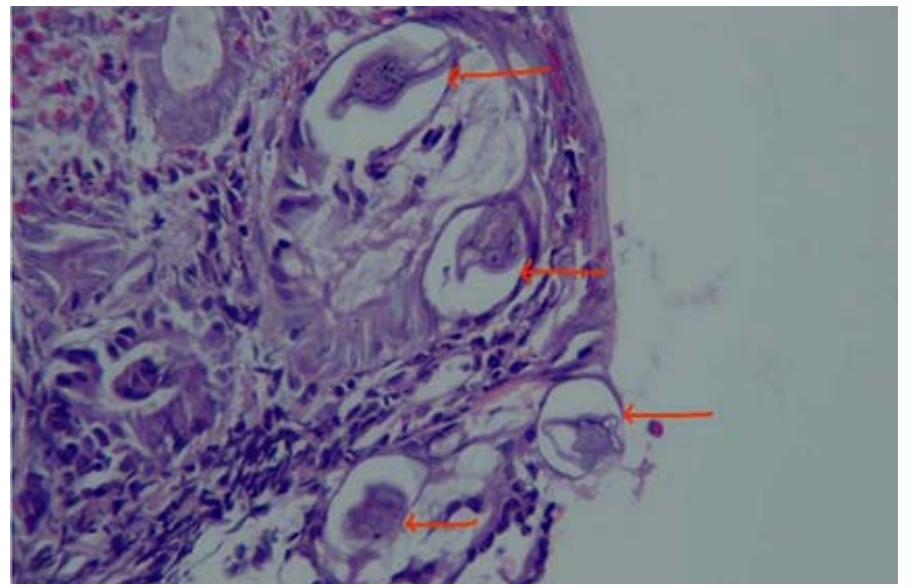

Patient was treated with albendazole orally $400 \mathrm{mg}$ B.D for 7 days. After getting anti helminthic, patient had dramatic improvement of general well-being. His vomiting subsided, appetite improved and leg edema gradually disappeared. After two weeks, patient gained $3 \mathrm{~kg}$ of body weight and repeat stool examination found no larvae or ova. We repeated upper GIT endoscopic examination and showed completely normal. (Figure 3) Patient was discharged home after a total 24 days of hospitalization.

Figure 3: Endoscopy of upper GIT showing marked improvement after treatment.

\section{Before treatment}

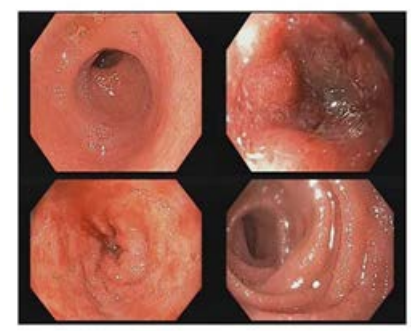

After treatment

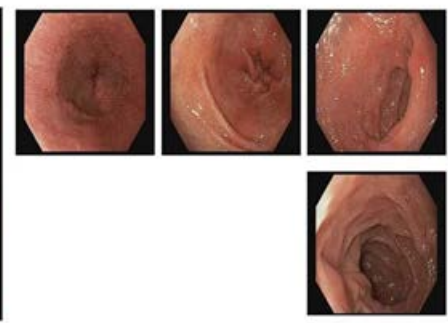

\section{DISCUSSION}

Strongyloides stercoralis is a common intestinal parasite in tropical and subtropical countries. ${ }^{8}$ To the best of our knowledge only few cases of duodenitis due to strongiloides stercoralis have been reported in Bangladesh. ${ }^{9,10} \mathrm{~S}$. stercoralis is a complex and unique nematode because it completes its entire life cycle both in free living and parasitic stages within the human host. Adult females, embedded in the intestinal mucosa, lay eggs that hatch internally. Rhabditiform larvae are excreted into stool and develop into filariform larvae in the soil. Humans are infected transcutaneously when filariform larvae penetrate intact skin (usually through the feet when people walk barefoot) migrate to the lungs via venous circulation. They penetrate the alveoli, ascend through the tracheobronchial tree, and are swallowed. After reaching GI tract, the larvae mature into adult females, reside in the duodenal and jejunal mucosa, and lay eggs. Eggs hatch into rhabditiform larvae, which are either passed in stools or penetrate intestinal mucosa or the perineal skin area. When rhabditiform larvae can develop directly into filariform larvae without leaving the host, this maturation results in autoinfection and continuation of parasitism causing autoinfection. . $^{3,8,11}$

The clinical syndromes of strongyloidiasis comprise a wide spectrum ranging from asymptomatic, acute or chronic infection to hyperinfection or dissemination. Strongyloidiasis is often asymptomatic in $50 \%$ of the cases. Chronic strongyloidiasis is mainly asymptomatic with occasional mild pulmonary and gastrointestinal symptoms. The hallmark of hyperinfection is development or exacerbation of gastrointestinal and pulmonary symptoms and increase numbers of larvae in stool and/or sputum. Pulmonary symptoms are fever, cough and dyspnea. Gastrointestinal symptoms include abdominal bloating, abdominal cramps, diarrhea, vomiting, weight loss, small bowel obstruction, paralytic ileus, perforating peptic ulcers and hematochezia or melena. ${ }^{12}$ In our case, the patient presented with intractable vomiting, dyspepsia, anorexia with significant weight loss, features of malnutrition with leg edema as a consequence of hypoalbuminemia but did not have any pulmonary symptoms.

Infection with strongyloides stercoralis is particularly important in immunocompromised patients receiving immunosuppressive drugs such as corticosteroids and chemotherapy for cancer and hemato- 
logic malignancies and also in those with organ transplantation, human T lymphotropic virus type 1, and HIV infections. Under such immunocompromising conditions the filariform larvae can be disseminated by autoinfection and progress to hyperinfection syndrome or disseminated

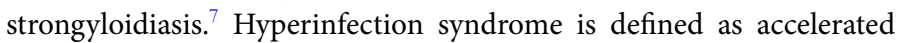
autoinfection with increased reproduction leading to excessive worm burden, whereas disseminated disease refers to the massive migration of infective larvae outside of the usual route after invading the gut wall to various organs, including liver and Central Nervous System (CNS). ${ }^{3}$ In our case, the patient received corticosteroid for 3 month in high dose which may be the cause of hyperinfection in the background of chronic asymptomatic carrier stage.

Eosinophilia may be the only sign, during the asymptomatic stage but eosinophilic count is not a reliable indicator of parasitic infections. Eosinophil count may be normal in hyperinfection or disseminated disease or patient on corticosteroid therapy. ${ }^{14}$ In case of our patient eosinophil count was not high, possibly due to high dose corticosteroid therapy.

Definitive diagnosis of strongyloidiasis is generally made based on detection of larvae in stool, sputum, and duodenal fluid/biopsy. However, strongyloidiasis is difficult to diagnose only on stool examination as the parasite load is low and the larval output is irregular in the majority of patients. ${ }^{14}$ Although most studies focus on finding the parasite by means of stool examination, single stool examinations are unrewarding in up to $50 \%$ to $70 \%$ of cases. ${ }^{15}$ Even after three stool examinations, positivity does not exceed $46 \% .{ }^{15,16}$ Endoscopic evaluation has been recognized to be an important tool for diagnosing strongyloidiasis, as worms and their maturing larvae colonize in the duodenum. ${ }^{7}$ Thus, this procedure is the most sensitive diagnostic technique for strongyloidiasis, with a false negativity less than $10 \% .{ }^{16}$ In our case initially endoscopic biopsy followed by stool microscopy had evidence of strongyloidiasis. Various immunodiagnostic assay tests have been used over the years, including indirect immunofluorescence analysis of fixed larvae and gelatin particle agglutination. It is shown in population based studies that stool examination generally underestimates the prevalence of S. stercoralis infection, whereas serological studies overestimate it. ${ }^{8}$ In our case no serological test was done.

Thiabendazole, albendazole and Ivermectin are the treatment of choice for strongiloidiasis and quite effective in most of the cases. Our patient was treated with albendazole.

\section{CONCLUSION}

Patient with immunocompromised condition are at risk of suffering from strongyloides stercoralis hyperinfection or disseminated disease. Physicians should be aware of these unusual manifestations of strongyloidiasis. Through this case report, we intend to emphasize the importance of doing endoscopy and endoscopic biopsy keeping in mind the stercoralis hyperinfection or disseminated disease when investigating the etiology of gastrointestinal symptoms in immunocompromised patients. Unless there is a high index of suspicion, strongyloidiasis, a readily treatable disease, may be missed.

\section{CONFLICTS OF INTEREST}

None.

\section{REFERENCES}

1. Grove DI. Historical introduction. In: Grove DI (editor), Strongyloidiasis: a major roundworm infection of man. Philadelphia: Taylor \& Francis; 1989;1-9.

2. Website: http://www.cdc.gov/parasites/strongyloides/health_professionals/2016.

3. Segarra-Newnham M. Manifestations, diagnosis and treatment of Strongyloides stercoralis infection. Ann of Pharmacother. 2007; 41(12): 1992-2001. doi: 10.1345/aph.1K302

4. Kim J, Joo H, Kim D, Lim H, Kang Y, Kim M. A case of gastric strongyloidiasis in a Korean patient. Korean J Parasitol. 2003; 41(1): 63-67. doi: $10.3347 / \mathrm{kjp} .2003 .41 .1 .63$

5. Shafaghi A, AskariK, Hajizadeh H, Mansou r-Ghanaei F. Gastric strongyloidiasis as multiple small gastric nodules. Am J Case Rep. 2012; 13: 7-10. doi: 10.12659/AJCR.882279

6. Kakati B, Dang S, Heif M, Caradine K, McKnight W, Aduli F. Strongyloides duodenitis: case report and review of literature. J Natl Med Assoc. 2011; 103: 60-63. doi: 10.1016/s0027-9684(15)30246-7

7. Kishimoto K, Hokama A, Hirata T, Ihama Y, Nakamoto M, Kinjo $\mathrm{N}$, et al. Endoscopic and histopathological study on the duodenum of Strongyloides stercoralis hyperinfection. World J Gastroenterol. 2008; 14: 1768-1773. doi: 10.3748/wjg.14.1768

8. Siddiqui AA, Berk SL. Diagnosis of Strongyloides stercoralis infection. Clin Infect Dis. 2001; 33: 1040-1047. doi: 10.1086/322707

9. Raihan ASMA, Miah RMA, Majumder TK, Rahman MM, Hasan M. Severe strongyloidiasis-a case report. Bangladesh J Medicine. 2005; 16: 93-95.

10. Khaled A, Ara N, Choudhry LL, Nasir TA. Strongyloidiasis in gastrointestinal biopsy. Pulse. 2009; 3(1): 27-28. doi: 10.3329/pulse.v3i1.6551

11. Concha R, Harrington WJr, Rogers AI. Intestinal strongyloidiasis: recognition, management, and determinants of outcome. J Clin Gastroenterol. 2005; 39(3): 203-211. doi: 10.1097/01.mcg.0000152779.68900.33

12. Amornvipas P, Nattee P, Chongsrisawat V, Pongpunlert W, Wisedopas PY. Overwhelming stronyloidiasis. Asian Biomedicine. 2009; 3(5): 531-536.

13. Agrawal V, Agrawal T, Ghoshal UC. Intestinal strongyloidiasis: A diagnosis frequently missed in the tropic. Trans R Soc Trop Med Hyg. 2009; 103(3): 242-246. doi: 10.1016/j.trstmh.2008.08.009

14. Ardic N. An overview of Strongyloides stercoralis and its infections. Mikrobiyol Bul. 2009; 43(1): 169-177.

15. Martín-Dávila P, Fortún J, López-VélezR, Norman F, Montes de OcaM, Zamarrón P, et al. Transmission of tropical and geographically restricted infections during solid-organ transplantation. Clin Microbiol Rev. 2008; 21(1): 60-96. doi: 10.1128/CMR.00021-07

16. Ganesh S, Cruz RJ Jr. Strongyloidiasis: A multifaceted disease. Gastroenterol Hepatol (N Y). 2011; 7: 194-196. 